\title{
Distributed Quantum Computation over Noisy Channels
}

\author{
J. I. Cirac ${ }^{1}$, A. K. Ekert ${ }^{2}$, S. F. Huelga ${ }^{3}$ and C. Macchiavello ${ }^{4}$ \\ ${ }^{1}$ Institut für Theoretische Physik, Universität Innsbruck, Technikerstrasse 25, A-6020, Innsbruck, Austria. \\ ${ }^{2}$ Department of Physics, Clarendon Laboratory, University of Oxford, Oxford OX1 3PU, U. K. \\ ${ }^{3}$ Optics Section, The Blackett Laboratory, Imperial College, London SWr 2BZ, U. K. \\ ${ }^{4}$ Dipartimento di Fisica "A. Volta" and INFM - Unità di Pavia, Via Bassi 6, 27100 Pavia, Italy.
}

We analyse the use of entangled states to perform quantum computations non locally among distant nodes in a quantum network. The complexity associated with the generation of multiparticle entangled states is quantified in terms of the concept of global cost. This parameter allows us to compare the use of physical resources in different schemes. We show that for ideal channels and for a sufficiently large number of nodes, the use of maximally entangled states is advantageous over uncorrelated ones. For noisy channels, one has to use entanglement purification procedures in order to create entangled states of high fidelity. We show that under certain circumstances a quantum network supplied with a maximally entangled input still yields a smaller global cost, provided that $n$ belongs to a given interval $n \in\left[n_{\min }, n_{\max }\right]$. The values of $n_{\min }$ and $n_{\max }$ crucially depend on the purification protocols used to establish the $n$ - processor entangled states, as well as on the presence of decoherence processes during the computation. The phase estimation problem has been used to illustrate this fact.

\section{INTRODUCTION}

Consider a quantum computation which can be divided into subroutines so that each subroutine can be run on a separate quantum processor. The processors may be placed at different locations/nodes of a computational network and may exchange data with a selected central processor [1]. Each processor operates on a partial input which has a fixed size. The partial inputs may be independent of each other, correlated or even entangled. When the computation is finished, the central processor, after collecting partial outputs from the other processors, stores the global output. This type of distributed computation may be repeated several times to yield a desired result and as such it features frequently in quantum parameter estimation procedures e.g. the phase estimation in frequency standards [2,3]. In some computational tasks, e.g. estimating a given parameter with a prescribed precision, the number of repetitions depends on the form of the input state - some entangled states require less repetitions than uncorrelated inputs. In the case of correlated input states, we have to pre-compute the input state for each run of the computation and this involves an additional use of physical resources. Are we still better off when the complexity of the pre-computation is included? How shall we include and compare the use of different physical resources?

In this paper we quantify this complexity by introducing the notion of cost of physical operations, such as the cost of establishing an entangled pair over a channel, the cost of transmitting one classical bit between components, the cost of running a quantum processor, etc. and discuss the performance of the distributed quantum computation when the inter-processor quantum communication is prone to errors, i.e. when the quantum channels among the processors are noisy.

This paper has been organized as follows. In section II we introduce the notion of cost in distributed quantum computation and show that if the ratio between the number of repetitions for the entangled and separate inputs decreases fast enough with the size of the network then, above some critical size, the computation can be made cheaper using entangled inputs. This behaviour is illustrated explicitly in the case of estimation of a small phase shift for qubits, which was considered in Ref. [1]. In section III we introduce the phase estimation problem for disentangled and maximally entangled states and show how to compare the two scenarios. In section IV we analyse the ideal case of noiseless channel and error-free computations. Noise along the channels linking the nodes of the network is taken into account in section $\mathrm{V}$ and the use of different purification schemes is considered. We show that for certain purification protocols it is advantageous to use entangled states, whereas for others the cost of the precomputation is not off-set by the subsequent reduction in the number of repetitions. In section VI we analyse the effects of decoherence during the computational process at each node and show how they affect the results obtained in the preceding sections. Finally, in section VII we summarise the main results of this work. 


\section{COSTS OF DISTRIBUTED QUANTUM COMPUTATION}

Let us start out by considering a generic scenario for distributed quantum computation: a central processor $A$ and $n-1$ processors labelled $B_{i}(i=1,2, \ldots, n-1)$ represent the nodes of a quantum network. These nodes agree on performing a given computation which consists of three steps: (1) Precomputation: In order to prepare the initial state of all the nodes they exchange certain classical and quantum information; (2) Computation at each node: Each of the nodes performs a well defined operation locally, followed by a measurement; (3) Communication of the results: The $B$ nodes report the outcomes of their measurements to the central node by sending $k$ bits of classical information. With this information, the central node estimates the outcome of the complete computation. The computation gives the correct result with certain probability, and therefore it has to be repeated a number of times in order to achieve a prescribed precision.

We are interested in the cost of the computation in terms of the number of uses of the processors, and the amount of classical and quantum communication involved in the whole computation. Let us denote by $P(n)$ the cost of the precomputation, i.e., the cost of establishing the initial state for the $n$ nodes. In general, if one wants to create an entangled state of all the nodes one has to send quantum information through the channels. Besides, due to the presence of noise during transmission and processing, the nodes will have to use either purification or error correction methods, which will require in addition some classical communication. Thus, the cost of precomputation will depend on the costs of sending qubits and classical bits through the communication channels that link the nodes. We denote by $Z$ the cost of running a quantum processor at each node, and by $Y$ the cost of sending the outcomes of the measurement from one node to the central node. Finally, we will denote by $R(n)$ the number of times the computation has to be repeated in order to obtain a prescribed precision. With these definitions, one can calculate the total cost of the computation. We wish to analyze the advantages of using entangled input states in the computation with respect to the case of initial uncorrelated states. Thus, we consider the following two scenarios:

(1) Disentangled states: If the initial state of the processors is disentangled, no communication is required in the precomputation. Therefore we take $P(n)=0$ [4] and obtain

$$
C_{1}(n)=R_{1}(n)[n Z+(n-1) Y] .
$$

(2) Entangled states: If the initial state of the processors is entangled, communication is required in the precomputation. We have

$$
C_{2}(n)=R_{2}(n)\left[P_{2}(n)+n Z+(n-1) Y\right]
$$

We can now evaluate the ratio between the cost $C_{2}(n)$ corresponding to entangled inputs and the cost $C_{1}(n)$ for independent processors. We obtain

$$
\frac{C_{2}(n)}{C_{1}(n)}=\frac{R_{2}(n)}{R_{1}(n)} \frac{P_{2}(n)+(n-1) Y+n Z}{(n-1) Y+n Z} .
$$

This ratio depends crucially on the ratio between the repetitions needed in each case, as well as on the cost of the precomputation. Under ideal conditions, the use of entangled states in general decreases the number of repetitions required, i.e. $R_{2}(n)<R_{1}(n)$. The use of entangled states will be cost efficient if what one gains in the number of repetitions compensates what one loses in the precomputation. Thus, we expect that there will be a certain $n_{m i n}$ such that if $n>n_{\text {min }}$, the use of entangled states is cost efficient. On the other hand, in the non ideal situation in which one has noise either in the quantum channel or during the computation, $R_{2}(n)$ may increase with $n$ more rapidly than $R_{1}(n)$ since entangled states are more prone to errors. Thus, we would expect that for a specific task there is a maximum value $n_{\max }$ such that for $n<n_{\max }$ the use of entangled states is cost efficient. Therefore, if $n_{\min }<n_{\max }$ there will be an interval $\left[n_{\min }, n_{\max }\right]$ in which entangled states are more advantageous. In the opposite case $n_{\min }>n_{\max }$, it will be more convenient to use disentangled states. The values of $n_{\min }$ and $n_{\max }$ will depend on the specific computation and of the methods used to create the entangled states. In the following we will concentrate on the specific but relevant problem of phase estimation addressed in Ref. [1]. We will first analyze the ideal noiseless case. Then we will consider the presence of noise in the quantum channel, and analyze two ways of overcoming this noise using entanglement purification. Finally, we will consider the effects of decoherence during the computation.

\section{THE PHASE ESTIMATION PROBLEM}

As an illustrative and, in spite of its simplicity, important example, consider a network of $n$ processors each performing computation $\mathcal{C}$ defined as a small conditional phase shift on a qubit 


$$
\begin{aligned}
& |0\rangle \longrightarrow|0\rangle, \\
& |1\rangle \longrightarrow e^{i \phi}|1\rangle .
\end{aligned}
$$

Computation $\mathcal{C}$ is performed at each of the $n$ nodes $\left(A, B_{i}\right)$. Each run consists of a conditional phase shift and the subsequent measurement protocol. Computation $\mathcal{C}$ is then reset after each repetition: we assume that no extra-phase accumulation is allowed by means of consecutive runs of the computation $\mathcal{C}$ on the same qubit before the measurement is performed.

\section{A. Disentangled states}

Without inter-node entanglement, the best way to estimate $\phi$ is to prepare each node in the initial state

$$
|\Psi\rangle_{i}=\frac{1}{\sqrt{2}}\left(|0\rangle_{i}+e^{-i \phi_{1}}|1\rangle_{i}\right) .
$$

where $\phi_{1}$ is a given phase that can be adjusted from computation to computation. Computation $\mathcal{C}$ is then applied, followed by a Hadamard transformation $H$ given by $H=\frac{1}{\sqrt{2}}(|0\rangle\langle 0|-| 1\rangle\langle 1|+| 0\rangle\langle 1|+| 1\rangle\langle 0|)$. The last step is the independent measurement of each qubit in the computational basis. The result of the measurement will be either 0 or 1 with probabilities $p_{1}$ and $1-p_{1}$, respectively. Each of the $B_{i}$ nodes then transmits one classical bit, corresponding to the result of the measurement, to the central node $A$. This process is repeated $R_{1}$ times yielding a binomial probability distribution. In this way one can estimate $\phi$ at node $A$ with precision

$$
\epsilon_{1}=\frac{\sqrt{\Delta p_{1}}}{\left|\frac{d p_{1}}{d \phi}\right| \sqrt{R}_{1}}
$$

where $\Delta p_{1}=p_{1}\left(1-p_{1}\right)$ is the variance of the binomial distribution. In general, $\epsilon_{1}$ will depend on $\phi$ and $\phi_{1}$. As soon as the first outcomes of the measurements are obtained (first repetitions) the value of $\phi_{1}$ can be adjusted in order to minimise $\epsilon_{1}[5]$.

\section{B. Entangled states}

Let us now assume that the initial state of the $n$ nodes is an entangled state of $n$ qubits of the form

$$
\left|\Psi_{i d}\right\rangle=\frac{1}{\sqrt{2}}\left(|000 \ldots 0\rangle+e^{-i n \phi_{2}}|111 \ldots 1\rangle\right) .
$$

Under ideal conditions, this state can be obtained as follows. The central processor at the central node generates $n-1$ EPR pairs and sends one member of each pair to the remaining nodes. An EPR pair shared between node $A$ and $B_{i}$ is referred to as the $A-B_{i}$ pair. In order to obtain the state in Eq. (8) we pick up one of the $n-1$ qubits at node $A$ and using it as a control qubit we apply the quantum controlled-NOT operation $\left|\epsilon_{1}\right\rangle\left|\epsilon_{2}\right\rangle \rightarrow\left|\epsilon_{1}\right\rangle\left|\epsilon_{1} \oplus \epsilon_{2}\right\rangle\left(\epsilon_{1,2}=0,1\right.$ and $\oplus$ denotes addition modulo 2 ) with the remaining $n-2$ target qubits at node $\mathrm{A}$. Then we measure the $n-2$ targets in the computational basis. At this stage we have already established an entangled state of all $n$ nodes; in order to put it into the form (8) we simply perform operation NOT $\left(\sigma_{x}\right)$ at location $B_{k}$ if the result of the measurement performed at $A$ on the qubit belonging to the $A-B_{k}$ pair was 1 . Finally, all the nodes perform a phase shift transformation with angle $\phi_{2}$. In Figure 1 we have depicted a set-up for the simplest case, involving only three processors. Once EPR pairs of the form $\frac{1}{\sqrt{2}}\left(|0\rangle_{A}|0\rangle_{B_{i}}+|1\rangle_{A}|1\rangle_{B_{i}}\right.$ ) have been established between nodes $A B_{1}$ and $A B_{2}$ (via channels represented in the figure by a thick line networking the central node with the other two), the central node $A$ performs a CNOT operation between the two qubits stored in $A$. A measurement of the target bit in the computational basis reduces the three-node composite state to either the state $\frac{1}{\sqrt{2}}\left(|0\rangle_{A}|0\rangle_{B_{1}}|0\rangle_{B_{2}}+|1\rangle_{A}|1\rangle_{B_{1}}|1\rangle_{B_{2}}\right.$ ) (outcome 0) or to the state $\frac{1}{\sqrt{2}}\left(|0\rangle_{A}|0\rangle_{B_{1}}|1\rangle_{B_{2}}+|1\rangle_{A}|1\rangle_{B_{1}}|0\rangle_{B_{2}}\right.$ ) (outcome 1). In the latter case, node $B_{2}$ has to invert the state of its qubit by means of a local operation. Therefore, the pre-computation requires nodes $A$ and $B_{2}$ to exchange classical information, as illustrated in the figure by the dotted line connecting those nodes.

Once a state close to the ideal state (8) is prepared, at each node we run computation $\mathcal{C}$ followed by the Hadamard transform. A measurement on the computational basis is then performed at each node. Nodes $B_{i}$ report their outcomes to node $A$ by broadcasting one bit of information and the overall parity of the reported bits and the outcome at node 
$A$ is calculated at $A$. This will give bit value 0 or 1 with probabilities $p_{2}(\phi)$ and $1-p_{2}(\phi)$. The procedure is repeated $R_{2}$ times and gives estimation of $\phi$ with precision

$$
\epsilon_{2}=\frac{\sqrt{\Delta p_{2}}}{\left|\frac{d p_{2}}{d \phi}\right| \sqrt{R}_{2}}
$$

where $\Delta p_{2}=p_{2}\left(1-p_{2}\right)$. In general, $\epsilon_{2}$ will depend on $\phi$ and $\phi_{2}$. As soon as the first outcomes of the measurements are obtained (first repetitions) the value of $\phi_{2}$ is adjusted in order to minimise $\epsilon_{2}$ [5].

\section{Comparison}

In order to compare the two procedures, we impose that the precision required is the same, i.e. $\epsilon_{1}=\epsilon_{2} \equiv \epsilon$. We obtain

$$
\frac{R_{2}(n)}{R_{1}(n)}=\frac{\Delta p_{2}}{\Delta p_{1}}\left(\frac{d p_{1}}{d \phi}\right)^{2}\left(\frac{d p_{2}}{d \phi}\right)^{-2}
$$

where in this expression the phases $\phi_{1,2}$ are assumed to be chosen independently of each other in order to minimize $\epsilon$. Once the values of $\Delta p_{i}$ and $d p_{i} / d \phi$ are known, one can substitute this expression in (3). The value of $P_{2}(n)$ will depend on the purification procedures used in the generation of the state (8).

\section{IDEAL CHANNELS AND COMPUTATIONS}

We consider first the simple situation in which no decoherence is present. In this case

$$
\begin{aligned}
& p_{1}=\frac{1}{2}\left[1-\cos \left(\phi-\phi_{1}\right)\right], \\
& p_{2}=\frac{1}{2}\left[1-\cos \left(n \phi-n \phi_{2}\right)\right] .
\end{aligned}
$$

One obtains $\epsilon_{1}=1 /\left(n R_{1}\right)^{1 / 2}$ and $\epsilon_{2}=1 /\left(n R_{2}\right)^{1 / 2}$ independently of the values of $\phi_{1,2}$. Therefore, we have $R_{1}(n)=$ $1 /\left(n \epsilon^{2}\right)$ and $R_{2}(n)=1 /(n \epsilon)^{2}$. On the other hand, the cost of the precomputation using the procedure described above is simply $P_{2}(n)=(n-1) X+(n-2) Y$, where $X$ is the cost of sending one qubit from the central node to any other node. We finally obtain

$$
\frac{C_{2}}{C_{1}}=\frac{1}{n}\left(\frac{(2 n-3) Y+(n-1) X+n Z}{(n-1) Y+n Z}\right) .
$$

This expression implies that for $n$ larger than a certain value $n_{\text {min }}$, the global cost for computation with entangled states is smaller than the one with independent states. It can be easily checked that for $(2 Y+X+Z) /(Y+Z) \gg 1$

$$
n_{\min } \approx \frac{2 Y+X+Z}{Y+Z} .
$$

If the cost $Z$ is much smaller than $X$ and $Y$, and $Y$ is much smaller than $X$, the threshold value is given by the ratio of the costs of distributing entanglement and classical communication. Figure 2 illustrates this behaviour.

\section{NOISY CHANNELS}

We have seen in the preceding sections that the use of entanglement is cost efficient above a certain threshold in the number of nodes of a quantum network. However, this result holds under the assumption that the channels networking the nodes are ideal. In particular, this implies that ideal entangled states $\left|\Psi_{i d}\right\rangle$ can be distributed among the $n$ nodes. In reality, this will never be the case, and therefore one has to analyze what will happen for noisy channels. While creating the state $\left|\Psi_{i d}\right\rangle$ (using, for instance, the protocol exemplified in Fig. 1), there will be errors. The state will no longer be a pure state, but will rather be described by a density operator $\rho \neq\left|\Psi_{i d}\right\rangle\left\langle\Psi_{i d}\right|$. The closeness of this state to the ideal one is measured by the fidelity $F_{0}=\left\langle\Psi_{i d}|\rho| \Psi_{i d}\right\rangle$. This means that the number of repetitions 
$R_{2}(n)$ required to perform the computation to a prescribed precision will increase (since the probability distribution of obtaining the right outcome becomes worse). On the other hand, one may use entanglement purification in order to increase the value of $F_{0}$ and therefore to reduce the number of required repetitions. However, this requires a higher precomputation cost $P_{2}(n)$. In this Section we analyze this problem for two different purification protocols, specially suited for different situations. In order to focus on the role of noise along the channels, we will assume that all local operations (both the ones required for the establishment of the entangled states and the ones involved in the computation at each node) are error free.

We assume that using a two-qubit purification protocol and after $s$ steps one creates an entangled pair between nodes $A$ and $B_{i}$ with fidelity $F_{s} \lesssim 1$. Let us denote by $P_{1}(s)$ the cost required to create such a state. Once we have the entangled pairs we use the method described above (see Fig. 1) to create the entangled state among the $n$ nodes. Assuming that the pairs are in a Werner-like state, the fidelity of the $n$-qubit state will be $F_{n} \simeq F_{s}^{(n-1)}$. In order to estimate how the results are affected by noise, we consider for simplicity

$$
\rho=x_{n}\left|\Psi_{i d}\right\rangle\left\langle\Psi_{i d}\right|+\frac{1-x_{n}}{2^{n}} I
$$

where $F_{n}=x_{n}+\left(1-x_{n}\right) / 2^{n}$. If we perform the computation with this state instead of the ideal one we obtain

$$
p_{2}=\frac{x_{n}}{2}\left[1-\cos \left(n \phi-n \phi_{2}\right)\right]+\frac{1-x_{n}}{2} .
$$

Therefore, to estimate the parameter $\phi$ with precision $\epsilon$, the computation has to be run a number of times

$$
R_{2}(n)=\frac{1}{n^{2} \epsilon^{2}}\left(1+\frac{1-x_{n}^{2}}{x_{n}^{2} \sin ^{2}\left(n \phi-n \phi_{2}\right)}\right)
$$

which now depends on $\phi$ and $\phi_{2}$. We take the optimal choice of $\phi_{2}$, which gives

$$
R_{2}(n)=\frac{1}{n^{2} \epsilon^{2} x_{n}^{2}}
$$

and obtain the cost ratio

$$
\frac{C_{2}(n)}{C_{1}(n)}=\frac{1}{n x_{n}^{2}}\left(\frac{(n-1) P_{0}(s)+(2 n-3) Y+n Z}{(n-1) Y+n Z}\right)
$$

Taking into account that $x_{n} \simeq F_{n} \simeq F_{s}^{(n-1)}$ we see that for a sufficiently high value of $n$, this ratio will be as big as we please. This implies the existence of a value $n_{\max }$ such that for $n \gtrsim n_{\max }$ there is no gain in using entangled states. In fact, it may happen that there is no gain for any value of $n$. On the other hand, the value $n_{\max }$ will depend on $P_{0}(s)$ and $F_{s}$, i.e. on the specific purification procedure. Besides, as before, there will be a value $n_{\min }$ such that if $n \in\left[n_{\min }, n_{\max }\right]$ then entangled states can be cost efficient. We will illustrate these features for some specific purification protocols.

\section{A. Purification Scheme 1}

Let us assume that we can create pairs between node $A$ (central node) and node $B_{i}(i=1, \ldots, n-1)$ with fidelity $F_{0}$. For simplicity we assume that they are in a Werner state. We consider that local operations are perfect, and therefore one can use the purification procedures of Refs. [6,7]. One can easily show that after $s$ successful purification steps the fidelity will be

$$
F_{s} \leq 1-(2 / 3)^{s}\left(1-F_{0}\right) \epsilon_{0} .
$$

In order to calculate $P_{0}(s)$ we note that to obtain one pair of fidelity $F_{s}$ one uses up at least $2^{s-1}$ pairs, and performs in each of them at least one operation. Therefore, $P_{0}(s) \geq 2^{s-1} U$, where now $U$ denotes the joint cost per used pair. Upon substituting these expressions in (18) we see that unless $U$ is much smaller than the other costs or $F_{0} \simeq 1$, there is no gain at all by using entangled states. 


\section{B. Purification Scheme 2}

We will evaluate now the cost associated with pre-computation when the nodes are networked via photonic channels [8]. As in the previous scheme, the maximum fidelity resulting in this case, $F_{s}$, approaches 1 with the number $s$ of purification steps exponentially fast

$$
F_{s} \simeq 1-a^{s}\left(1-F_{0}\right)
$$

where $a<1$ is a constant. In this scheme the cost of establishing one entangled pair $P_{0}(s)=b s$ is proportional to the number of steps. Substituting these quantities in Eq. (18) one can see the existence of an interval $\left[n_{m i n}, n_{m a x}\right]$ in which the use of maximally entangled states is more convenient with respect to uncorrelated ones.

This purification protocol is more efficient than the previous one since $P_{0}$ does not scale exponentially with $s$. The reason for this scaling law in the purification scheme 1 is that one discards one pair at each purification step, which makes it very ineffective. Therefore, it is more desirable to use a purification scheme giving a finite yield, as the hashing or breeding methods [9]. Moreover, the purification protocols used here are just based on the ones developed for two qubits, which lead to the exponential dependence of $F$ on $n$. By using ideas similar to the ones developed for quantum repeaters [10] it may be possible to improve this exponential dependence.

\section{NOISE IN THE COMPUTATIONAL PROCESS}

Let us now assume that at each node the computation itself is not error-free but dephasing-type decoherence is present at a rate $g$, namely a random phase is introduced in front of the component $|1\rangle$ of the qubit with probability $e^{-g t}$ at time $t$ (notice that if one considers a quantum optical implementation, the results that we will present in the following hold also in the presence of spontaneous emission). When measured in the computational basis, the bit value 0 will now be obtained with probabilities [3]

$$
p_{1}=\frac{1}{2}\left[1-\cos \left(\phi-\phi_{1}\right) e^{-g t_{c}}\right]
$$

when dealing with independent processors and

$$
p_{2}=\frac{F}{2}\left[1-\cos \left(n \phi-n \phi_{2}\right) e^{-n g t_{c}}\right]+\frac{1-F}{2}
$$

in the case of maximally entangled nodes of the form (8). In the above equations $t_{c}$ is the time required to perform computation $\mathcal{C}$ and will be regarded as a fixed parameter in the comparison of the two schemes. To achieve resolution $\epsilon$ the computation $\mathcal{C}$ must be performed

$$
R_{1}=\frac{1}{n \epsilon^{2}} \frac{e^{2 g t_{c}}-\cos ^{2}\left(\phi-\phi_{1}\right)}{\sin ^{2}\left(\phi-\phi_{1}\right)}
$$

times with independent processors and

$$
R_{2}=\frac{1}{n^{2} \epsilon^{2} F^{2}} \frac{e^{2 n g t_{c}}-F^{2} \cos ^{2}\left(n \phi-n \phi_{2}\right)}{\sin ^{2}\left(n \phi-n \phi_{2}\right)}
$$

times when a maximally entangled input is distributed among the $n$ nodes. The relative cost of both procedures is no longer $\phi$ independent. Following the argument presented in the previous Section, we select the controllable phases $\phi_{1,2}$ in both procedures in such a way that the measured phase approaches $\pi / 2$ when dealing with uncorrelated inputs and $\pi / 2 n$ when one uses entangled states. Therefore, we can write

$$
R_{1}=\frac{1}{n \epsilon^{2}} e^{2 g t_{c}}
$$

while

$$
R_{2}=\frac{1}{n^{2} \epsilon^{2} F^{2}} e^{2 n g t_{c}}
$$

The cost ratio is then given by 


$$
\frac{C_{2}}{C_{1}}=e^{2 g t_{c}(n-1)}\left(\frac{C_{2}}{C_{1}}\right)_{g=0} .
$$

As it can be seen from Figure 3, the effect of dephasing type decoherence is negligible in the limit $g t_{c} \ll 1 / n$. The net effect of decoherence during the computational process is a further reduction of the domain where the use of entanglement is cost efficient, being $n_{\max }(g \neq 0)<n_{\max }(g=0)$.

\section{CONCLUSIONS}

In summary, we have introduced the notion of a generic cost of physical operations in distributed quantum computation. This parameter allows to quantify the efficiency of a quantum computation which can be run separately on different quantum processors belonging to a quantum network. Previous work [1] 11] has shown that the use of entangled states could be advantageous for certain computations. However, it was not obvious that the cost of generating entanglement or the inclusion of noise during the computational process, might not nullify their advantage. We have shown that under certain circumstances a quantum network supplied with a maximally entangled input yields a smaller global cost than the one required when dealing with $n$ independent inputs, provided that $n$ belongs to a given interval $n \in\left[n_{\min }, n_{\max }\right]$. We have illustrated this for the case of phase estimation. The values of $n_{\min }$ and $n_{\max }$ crucially depend on the purification protocols used to establish the $n-$ processor entangled states, as well as on the presence of decoherence processes during the computation.

We are grateful to C. H. Bennett, L. Hardy, R. Jozsa, N. Lütkenhaus, M. B. Plenio, S. Popescu and S. van Enk for helpful discussions.

This work was supported in part by the European TMR Research Networks ERP-4061PL95-1412 and ERPFMRXCT96066, Hewlett-Packard, The Royal Society of London and Elsag-Bailey, a Finmeccanica Company. SFH acknowledges support from DGICYT Project No. PB-95-0594 (Spain).

* $\quad$ Permanent Address: Departamento de Física. Universidad de Oviedo. Calvo Sotelo s/n 33007, Oviedo, Spain.

[1] L. Grover, Quantum Telecomputation, Report No. quant-ph/9704012.

[2] J.J. Bollinger, W.M. Itano, D.J. Wineland and D.J. Heinzen, Phys. Rev. A 54, R4649 (1996).

[3] S. F. Huelga, C. Macchiavello, T. Pellizzari, A. Ekert, M.B. Plenio and J.I. Cirac, Phys. Rev. Lett. 79, 3865 (1997).

[4] We could consider the cost of creating the initial disentangling state at this stage. In order not to overload the number of parameter and concentrate in the differences caused by the use of entanglement we will omit this cost. We will also omit here the cost of local operations, although they could be easily included. The conclusions of our analysis do not change if one includes these costs.

[5] D. J. Wineland et al. Phys. Rev. A 50, 67 (1994).

[6] C.H. Bennett, G. Brassard, S. Popescu, B. Schumacher, J.A. Smolin and W.K. Wootters, Phys. Rev. Lett. 76, 722 (1996).

[7] D. Deutsch, A. Ekert, R. Jozsa, C. Macchiavello, S. Popescu and A. Sanpera, Phys. Rev. Lett. 77, 2818 (1996).

[8] S.J. van Enk, J.I. Cirac and P. Zoller, Phys. Rev. Lett. 78, 4293 (1997); S.J. van Enk, J.I. Cirac and P. Zoller, Science 279, 205 (1998).

[9] C.H. Bennett, D.P. DiVincenzo, J.A. Smolin and W.K. Wootters, Phys. Rev. A 54, 3824 (1996).

[10] H.-J. Briegel, W. Dür, J.I. Cirac and P. Zoller, Phys. Rev. Lett. 81, 5932 (1998).

[11] H. Buhrman, R. Cleve and W. van Dam, Quantum entanglement and communication complexity, Report No. quant$\mathrm{ph} / 9705033$.

FIG. 1. Experimental set-up for performing a distributed quantum computation between three nodes sharing an entangled state.

FIG. 2. Cost ratio $C_{2} / C_{1}$ as a function of the number of nodes within the quantum network for two different sets of values of the parameters $(X, Y, Z)=(100,10,1),(1000,10,1)$. Above a threshold $n_{t h} \approx X / Y$, the global cost of the computation is smaller when using a maximally entangled input. 
FIG. 3. Cost ratio as a function of the number of nodes for a phase estimation problem. The dotted line corresponds to the case when dephasing type decoherence takes place during the computational process. The net effect is that the domain where the use of entanglement is cost efficient gets shrinked with respect to the case of error free computation, represented by the solid line. 
Figure 1

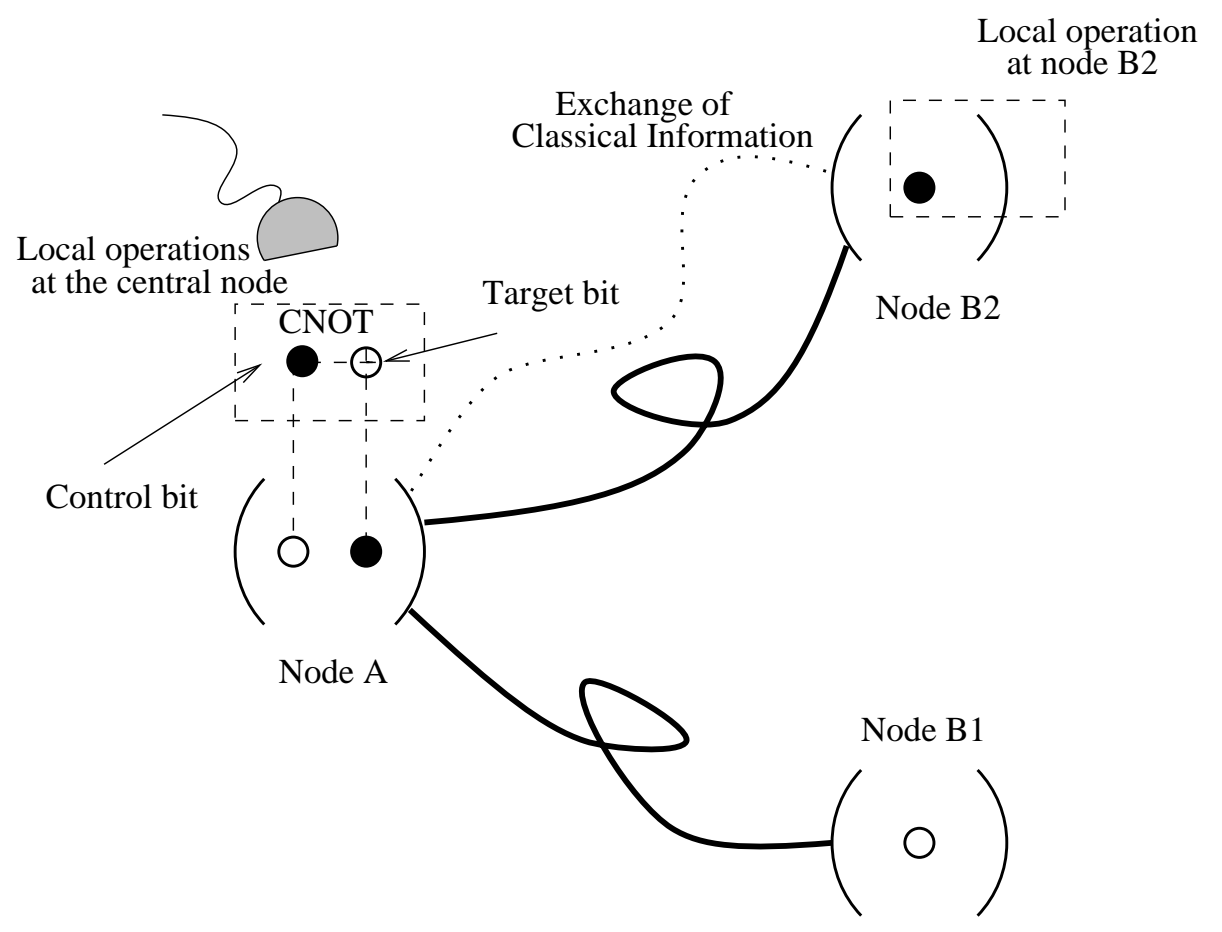


Figure 2

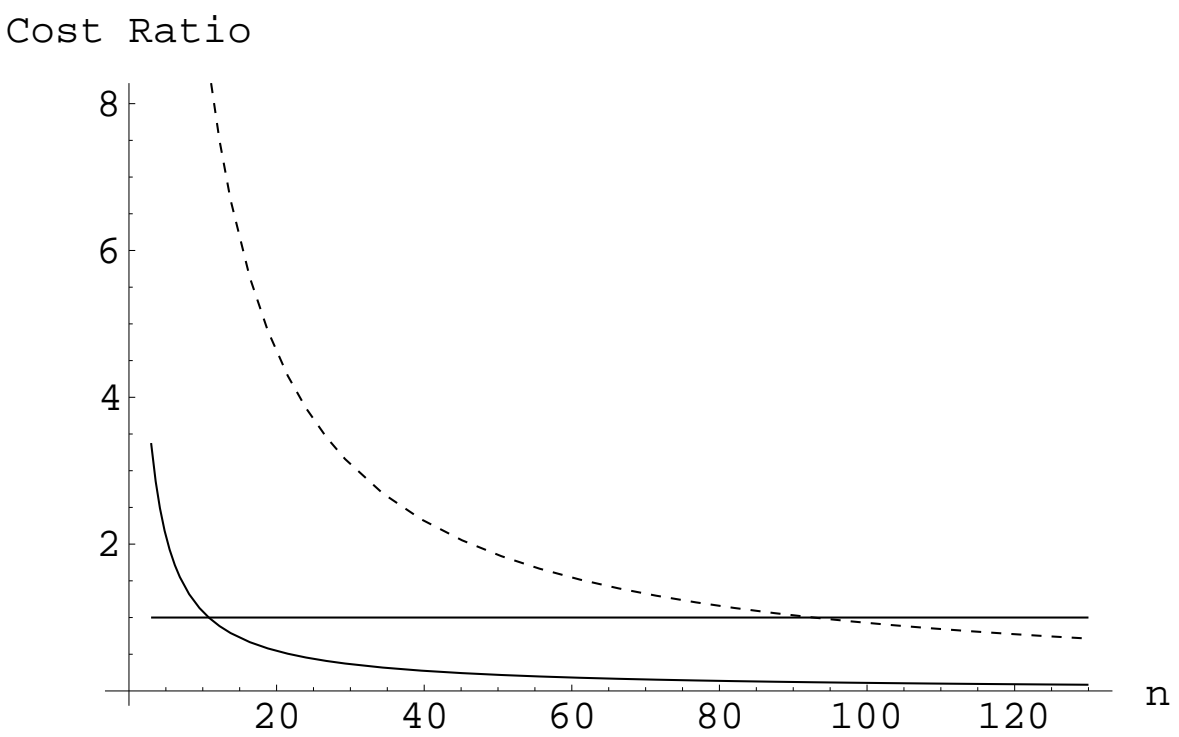


Figure 3

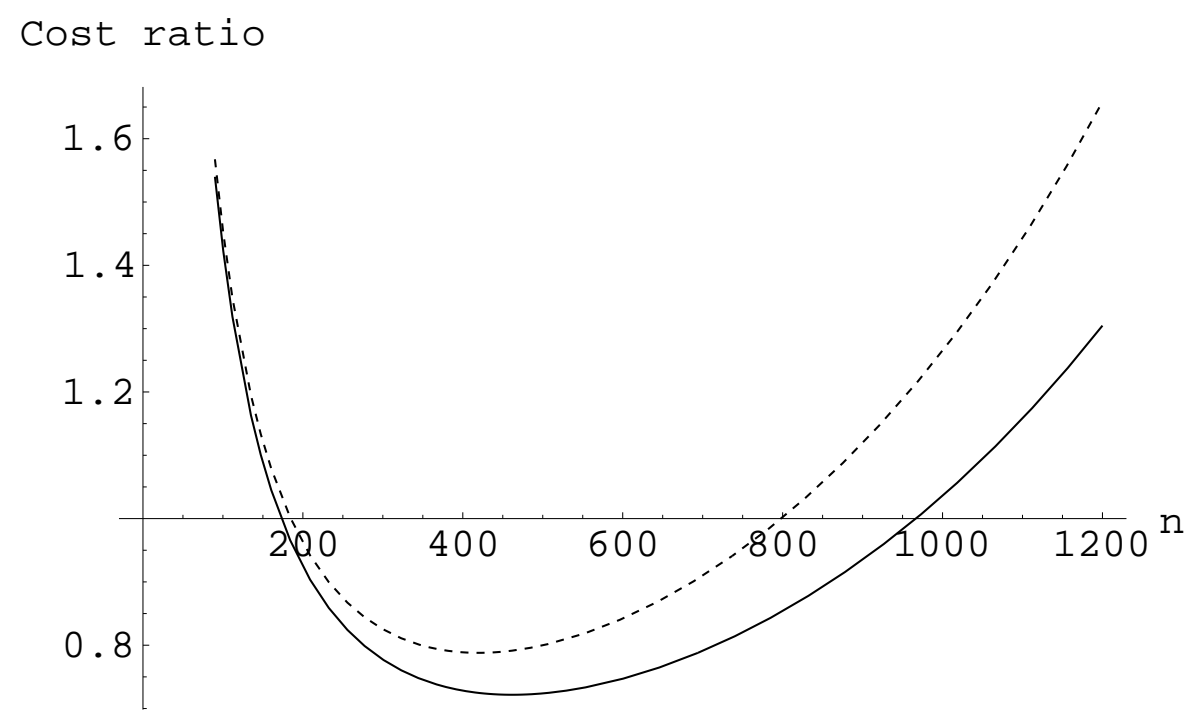

